\title{
Air Pollution Hotspot Detection and Source Feature Analysis using Cross-Domain Urban Data
}

\author{
Yawen Zhang \\ Department of Computer Science, \\ University of Colorado Boulder \\ Boulder, CO, USA \\ yawen.zhang@colorado.edu
}

\author{
Michael Hannigan \\ Department of Mechanical \\ Engineering, \\ Environmental Engineering Program, \\ University of Colorado Boulder \\ Boulder, CO, USA \\ michael.hannigan@colorado.edu
}

\author{
Qin Lv \\ Department of Computer Science, \\ University of Colorado Boulder \\ Boulder, CO, USA \\ qin.lv@colorado.edu
}

\begin{abstract}
Air pollution is a major global environmental health threat, in particular for people who live or work near air pollution sources. Areas adjacent to pollution sources often have high ambient pollution concentrations, and those areas are commonly referred to as air pollution hotspots. In this work, we explore the use of mobile sensing data to detect pollution hotspots. We propose a two-step approach to detect hotspots from unevenly sampled mobile sensing data. To contextualize the detected hotspots and discover potential pollution source characteristics, we explore a variety of cross-domain urban data and extract features from them for hotspot inference. Evaluation results using real-world mobile sensing air quality data as well as cross-domain urban data demonstrate the effectiveness of our approach in detecting and inferring pollution hotspots.
\end{abstract}

\section{CCS CONCEPTS}

- Information systems $\rightarrow$ Data mining; Spatial-temporal systems; • Applied computing $\rightarrow$ Environmental sciences.

\section{KEYWORDS}

Air pollution hotspot detection, pollution source feature analysis, cross-domain urban data

\section{ACM Reference Format:}

Yawen Zhang, Michael Hannigan, and Qin Lv. 2021. Air Pollution Hotspot Detection and Source Feature Analysis using Cross-Domain Urban Data. In 29th International Conference on Advances in Geographic Information Systems (SIGSPATIAL '21), November 2-5, 2021, Beijing, China. ACM, New York, NY, USA, 4 pages. https://doi.org/10.1145/3474717.3484263

\section{INTRODUCTION}

Air pollution is a major environmental problem and has adverse impact on people's health. People who live or work near air pollution sources, such as busy highways, or industries that emit air pollutants are at higher risk of negative health impact caused by pollutants [1]. For health impact assessment as well as air quality management, it is crucial to understand the spatial patterns of air

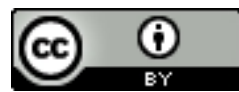

This work is licensed under a Creative Commons Attribution International 4.0 License. SIGSPATIAL '21, November 2-5, 2021, Beijing, China

(c) 2021 Copyright held by the owner/author(s).

ACM ISBN 978-1-4503-8664-7/21/11.

https://doi.org/10.1145/3474717.3484263 quality, especially areas with high ambient pollution concentrations, which are commonly referred to as air pollution hotspots [6].

Due to high variability of air pollutants in both space and time, it is infeasible to detect air pollution hotspots from observations of conventional sparse monitoring stations. As such, mobile sensing has been explored to observe air quality at high spatial resolution. Google equipped its Street View vehicles with air pollutant sensors to monitor street-by-street air quality such as black carbon (BC) and $\mathrm{NO}_{2}$ [7]. This new data modality enables the discovery of air pollution hotspots, in particular the fine-scale localized ones [2].

For air pollution hotspots, discovering their potential emission sources is of importance. There are numerous local emission sources distributed spatially, which are not well represented in the conventional emission inventories [2]. Figure 1 shows some of the representative air pollution hotspots we have detected from mobile sensing data. They are related to various types of emission sources, such as truck or car businesses, industrial areas, road intersections. The cross-domain urban data, such as Point-of-Interest (POI), land use, vehicle idling, and energy consumption data, provide a good representation of local emission sources and thus enable automatic association of air pollution hotspots with their plausible sources.

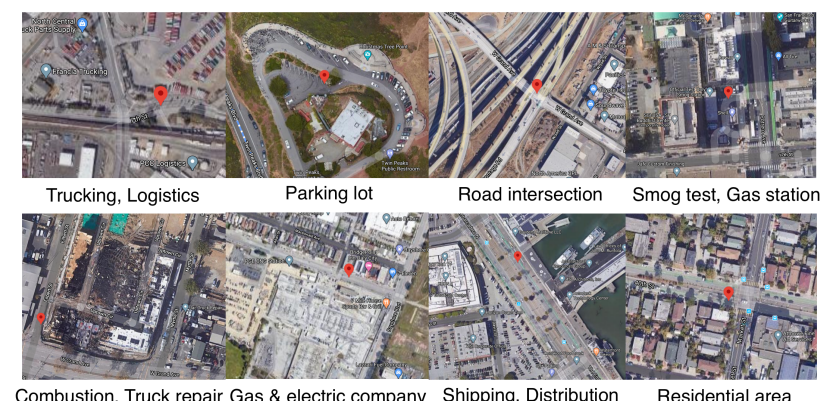

Figure 1: Representative air pollution hotspots and plausible neighborhood pollution sources (from Google Maps).

In this study, we propose to leverage mobile sensing and crossdomain urban data to detect air pollution hotspots and characterize local emission sources. More specifically, we develop a two-step approach to detect air pollution hotspots from mobile sensing data, which addresses the uneven sampling issue with mobile sensing. The discovery of hotspots help in narrowing down the regions of interest, which can be further investigated by domain experts and regulatory agencies. Our analysis of hotspots and source features yields useful insights regarding neighborhood pollution sources. 


\section{RELATED WORK}

Hotspot Detection. Spatial hotspot detection aims at discovering geographical regions with significantly higher concentration of activities. The technique for hotspot detection is usually designed based on study objective and data type (e.g., grid or network data). Different techniques have been proposed for hotspot detection, including local indicators of spatial association [8], Kernel Density Estimation (KDE) [9]. Most existing studies are conducted on uniformly sampled data, i.e., data that are collected evenly in both space and time. Our study proposes techniques to detect hotspots from unevenly sampled mobile sensing data.

Pollution Source Discovery. To analyze pollution sources, atmospheric scientists rely on receptor modeling [3], which leverages measurements of chemical composition data for gas and particle phase air pollutant samples collected at specific sites to quantify source contributions. Due to measurements required for modeling, they are conducted at a limited number of sampling sites and can only provide information regarding pollution sources at a relatively coarse granularity. To automate the process of discovering potential local pollution sources, this study explores the use of cross-domain urban data to study their relationship with pollution hotspots.

\section{METHODOLOGY}

\subsection{Overview}

The methodology consists of three key components: hotspot detection, source-related feature extraction, and hotspot inference.

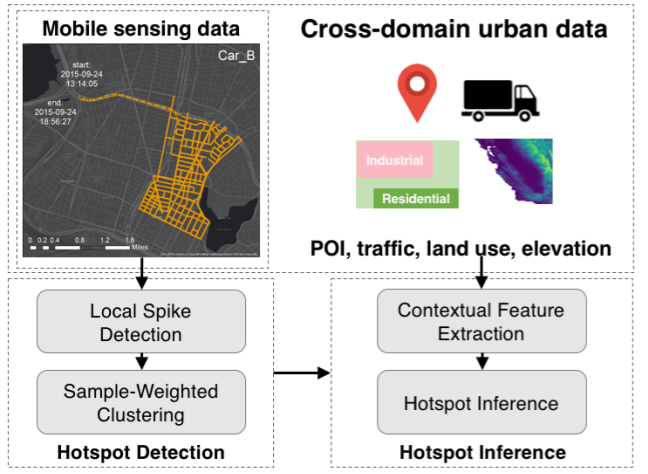

Figure 2: Overview of the methodology.

\subsection{Air Pollution Hotspot Detection}

Step 1: Local Spike Detection. This step detects spikes corresponding to locations where multipollutant concentrations exceed both background and neighboring ambient levels. For each day $D$, given air pollutant $a$ and car trajectory $\boldsymbol{p}_{1} \rightarrow \boldsymbol{p}_{2} \rightarrow \ldots \rightarrow \boldsymbol{p}_{n}$, we use a sliding window with varying size to extract segment $M$ of trajectory $\boldsymbol{p}_{m} \rightarrow \boldsymbol{p}_{m+1} \rightarrow \ldots \rightarrow \boldsymbol{p}_{m+o}, 1 \leq m \leq n-o$, where $o$ is the number of valid observations in $M$. Essentially, a segment $M$ should satisfy the following constraints:

$$
\begin{cases}\text { Pollution level: } & a_{M} \geq a_{D}, a_{M} \geq \max \left(r a_{L}, r a_{R}\right) \\ \text { Range: } & \boldsymbol{w}_{\min } \leq \operatorname{dist}\left(\boldsymbol{p}_{m}, \boldsymbol{p}_{m+o}\right) \leq \boldsymbol{w}_{\max }\end{cases}
$$

In pollution level constraint, $a_{M}$ denotes the median air pollutant concentration of segment $M . a_{D}$ and $\left(a_{L}, a_{R}\right)$ represent the background and neighboring pollution levels. Here, $a_{D}$ is computed as the median air pollutant concentration of all observations on day $D$, and $\left(a_{L}, a_{R}\right)$ are computed as the median air pollutant concentrations of the windows to the left and right of $M$. This constraint requires that $a_{M}$ not only exceeds the background pollution level $a_{D}$, but also exceeds at least $r$ of the neighboring pollution level $\left(a_{L}, a_{R}\right) . r(r \geq 1)$ denotes the ratio of $a_{M}$ to its neighbors $\left(a_{L}, a_{R}\right)$. In range constraint, $\operatorname{dist}\left(\boldsymbol{p}_{m}, \boldsymbol{p}_{m+o}\right)$ denotes the distance between the start and end point of M. Here, $w$ defines the size range of the sliding window, thus $\operatorname{dist}\left(\boldsymbol{p}_{m}, \boldsymbol{p}_{m+o}\right)$ should be within the range of $\boldsymbol{w}$, i.e., $\left[\boldsymbol{w}_{\min }, \boldsymbol{w}_{\max }\right]$.

Step 2: Sample-Weighted Clustering. Local spikes represent elevated pollutant concentrations, but some of them are not necessarily related to local pollution hotspots. For instance, some spikes are just outliers in observations, such as extremely high pollutant levels caused by encounters with other vehicles' exhaust plumes [2]. Here, we consider spike frequency $S_{\boldsymbol{g}}$ (i.e., total number of spikes detected in a city grid $\boldsymbol{g}$ ), and set a minimum threshold of $S_{\boldsymbol{g}}$ to remove city grids with only a few random spikes.

Given the uneven sampling issue with mobile sensing, it is likely that city grids with more repeated observations would have more spikes. For a fair comparison across city grids, we include another indicator - spikes' temporal hit rate (THR) - to quantify the persistence of hotspots. For a city grid $\boldsymbol{g}, T H R_{\boldsymbol{g}}$ is defined as follows:

$$
T H R_{\boldsymbol{g}}=\frac{\left|T_{S}(\boldsymbol{g}) \cap T_{O}(\boldsymbol{g})\right|}{|T(\boldsymbol{g})|}
$$

where $T(\boldsymbol{g})=\left[t_{1}, t_{2}, \ldots, t_{z}\right]$. For a specific hour $i$, if there is a valid data point, $t_{i}$ would be assigned to 1 , otherwise 0 . Here, $T(\boldsymbol{g})$ is computed separately for spikes and observations as $T_{S}(\boldsymbol{g})$ and $T_{O}(\boldsymbol{g})$, and their intersection indicates their temporal consistency. $|T(\boldsymbol{g})|$ denotes the total number of hours. Higher $T H R_{\boldsymbol{g}}$ indicates higher persistence of the spikes within grid $\boldsymbol{g}$.

In this step, we firstly aggregate all the spikes into city grids $\mathcal{G}$, and compute $S_{\boldsymbol{g}}$ and $T H R_{\boldsymbol{g}}$ for each city grid $\boldsymbol{g}$. These two indicators are used to measure the robustness and persistence of $\boldsymbol{g}$. Then, we detect hotspots by adapting the mean shift clustering algorithm [5]. This procedure is a natural way to identify pollution hotspots, i.e., a pollution source is usually located at a mode of KDE and it causes spikes within its radius- $b$ window. Let $s=($ lat, lon $)$ be the center of the current window, and $\mathcal{S}(s)$ be the data points inside the window. The mean shift vector would always lead to local density maximum. Here, we incorporate sample weights into the procedure, and the mean shift vector using a Gaussian kernel is computed according to the following equations:

$$
\begin{gathered}
m(s)=\frac{\sum_{s_{i} \in \mathcal{S}(s)} K\left(s_{\boldsymbol{i}}-\boldsymbol{s}\right) \boldsymbol{s}_{\boldsymbol{i}}}{\sum_{\boldsymbol{s}_{\boldsymbol{i}} \in \mathcal{S}(s)} K\left(\boldsymbol{s}_{\boldsymbol{i}}-\boldsymbol{s}\right)}, \\
K\left(\boldsymbol{s}_{\boldsymbol{i}}-\boldsymbol{s}\right)=W_{\boldsymbol{s}_{\boldsymbol{i}}} e^{\frac{-\left\|\boldsymbol{s}_{\boldsymbol{i}}-\boldsymbol{s}^{2}\right\|^{2}}{2 b^{2}}} \\
W_{\boldsymbol{s}_{\boldsymbol{i}}}=S_{\boldsymbol{g}} T H R_{\boldsymbol{g}}
\end{gathered}
$$

where $K(\cdot)$ is the kernel function, and sample weight $W_{s_{i}}$ is computed as the product of $S_{\boldsymbol{g}}$ and $T H R_{\boldsymbol{g}}$. By assigning weights to the grids, clusters are shifting towards grids that are more robust and persistent. We label city grids that form clusters as hotspots. 


\subsection{Source Feature Vector Representation}

3.3.1 Contextual Feature Extraction. We extract the following features and concatenate them to form source feature vector.

- POI features. POI features are extracted within a spatial range of $100 \mathrm{~m}$ of each hotspot. They were collected using Google Place API. The POI features include (1) number of POIs in each POI category, (2) number of keywords in POI names (a list of keywords related to pollution sources, and are not included in POI categories), (3) total number of POIs, and (4) POI entropy in terms of category.

- Land use features. For each hotspot, we compute its spatial distance to the nearest types of land use, such as industrial, commercial, and residential.

- Vehicle idling features. Vehicle idling data is provided by Geotab. The vehicle idling features include (1) cumulative, median, mean idling time, (2) \% of different types of vehicles, (3) mean idling time of each vehicle type, and (4)\% of different types of engine, such as gas or diesel.

- Elevation features. We compute mean, standard deviation, and concave index (using $3 * 3$ Laplacian filter) of elevation.

\subsection{Air Pollution Hotspot Inference}

To validate the effectiveness of source features, we build models to infer hotspots using source feature vectors.

\section{EVALUATION}

\subsection{Experimental Setup}

4.1.1 Datasets. We obtained the mobile sensing air quality data from Google. The air quality dataset in the city of Oakland is collected from June 2015 to May 2016 and has the densest coverage. In other areas of San Francisco (SF), the dataset is collected from May 2016 to August 2017. We focus on three key health-relevant air pollutants: $\mathrm{BC}, \mathrm{NO}$, and $\mathrm{NO} 2$. We partition geographic regions into 50 $\mathrm{m} \times 50 \mathrm{~m}$ city grids, and collect multi-source urban data including Google POIs, OpenStreetMap (OSM) land use data, Geotab vehicle idling statistics (153 m $\times 153 \mathrm{~m}$ grids), and SRTM 90-m elevation data. We use city grids as the basis for collecting and matching data at different spatial formats and/or resolutions.

4.1.2 Parameter Settings. The major parameters in hotspot detection include (1) $a_{D}$ : the background air pollution level, (2) $r$ : the minimum ratio of pollutant elevations compared to spatial neighbors, (3) $\boldsymbol{w}$ : size range of the sliding window, (4) minimum $S_{\boldsymbol{g}}$ : the minimum threshold of spike frequency for clustering, and (5) $b$ : the bandwidth for mean shift clustering. By default, we set $a_{D}$ to the daily median pollution levels, $r=1.5, w=[50,60, \ldots, 100]$ with an intercept of $10 \mathrm{~m}$, minimum $S_{\boldsymbol{g}}=10$, and $b=100 \mathrm{~m}$.

\subsection{Hotspot Detection Results}

4.2.1 Evaluation Metrics. We consider three performance metrics: mean elevated level of air pollution $(E A)$, robustness index $(R I)$, and mean temporal hit rate $(T H R)$. EA measures the ratio of hotspot pollution levels to the background levels, and is defined as:

$$
E A=\frac{\sum_{1}^{q} \frac{a_{g, i}}{a_{D, i}}}{q}, 1 \leq i \leq q
$$

where $a_{\boldsymbol{g}, i}$ denotes the pollutant concentration at hotspot $\boldsymbol{g}$, and $E A$ computes the mean elevated level of multiple pollutants. Higher $E A$ indicates higher elevated level. To quantify the robustness of hotspots, we randomly separate the raw datasets into two equalsized parts, and conduct hotspot detection on them. RI measures the robustness of the detected hotspots, and is defined as follows:

$$
R I=\frac{\frac{\left|\mathcal{H}_{k_{1}}(\boldsymbol{g}) \cap \mathcal{H}_{k_{2}}(\boldsymbol{g})\right|}{\left|\mathcal{H}_{k_{1}}(\boldsymbol{g}) \cup \mathcal{H}_{k_{2}}(\boldsymbol{g})\right|}}{h r}
$$

where $H_{k_{1}}(\boldsymbol{g})$ and $H_{k_{2}}(\boldsymbol{g})$ are the hotspots of the two parts, and $k_{1}$, $k_{2}$ are their number of hotspots. We use Jaccard index to measure similarity. The higher the Jaccard index, the more similar these two parts, and the more robust the detected hotspots. We compute $R I$ as the ratio of Jaccard index to the hotspot percentage $h r$, i.e., the average percentage of valid city grids that are detected as hotspots. The higher the RI, the more robust the hotspots. Moreover, we use mean THR to measure the persistence of hotspots. The higher the $T H R$, the more persistent the detected hotspots.

\subsubsection{Baseline Methods.}

- SDO: the spike detection only (SDO) method only performs spike detection and label all the spikes as hotspots.

- TNAS: the threshold on total number of aggregated spikes (TNAS) method performs spike detection first, and then applies a threshold on spike frequency. Those above the threshold are detected as hotspots.

4.2.3 Method comparison results. Our proposed approach achieves the best performance on all three metrics, demonstrating this approach can detect more robust and persistent hotspots. SDO detects much more hotspots but they are the least robust and persistent, which is consistent with the fact that there are a lot of short-lived spikes. Compared with TNAS, our approach adds THR into sample weights for clustering thus identify more persistent hotspots.

Table 1: Comparison of different methods.

\begin{tabular}{ccccc}
\hline Method & EA & RI & THR & \# hotspots \\
\hline SDO & 2.234 & 1.017 & 0.242 & 6521 \\
TNAS & 2.283 & 2.706 & 0.382 & 2357 \\
Our Approach & 2.303 & 4.540 & 0.424 & 1484 \\
\hline
\end{tabular}

\subsection{Empirical Analysis on Source Features}

Figure 3 shows the most representative features in each feature category to illustrate their effectiveness in discriminating hotspots and non-hotspots. In Figure 3a, compared with non-hotspots, hotspots have higher number of POIs in categories such as car repair, gas station, parking, moving company, meal takeaway, etc. We observe in Figure $3 \mathrm{~b}$ that hotspots have higher number of POIs with name keywords such as "trucking", "wheels", "smog", "towing", and "intersection". For land use features shown in Figure 3c, hotspots are closer to industrial and commercial areas than non-hotspots. As shown in Figure 3d, hotspots have much longer cumulative idling time than non-hotspots, which indicates that vehicle idling is very likely a contributor to some of the detected hotspots. In Figure 3f, we observe that hotspots have lower elevation than non-hotspots. Locations at lower elevation are more likely to trap air pollutants. 


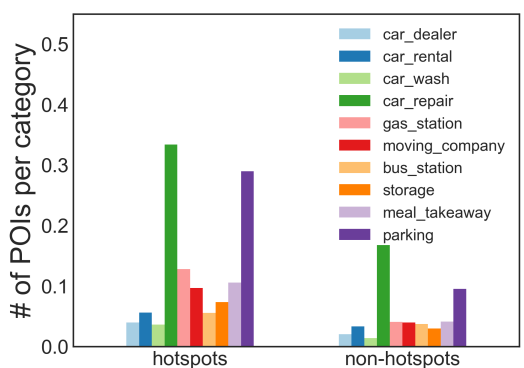

(a) POI categories

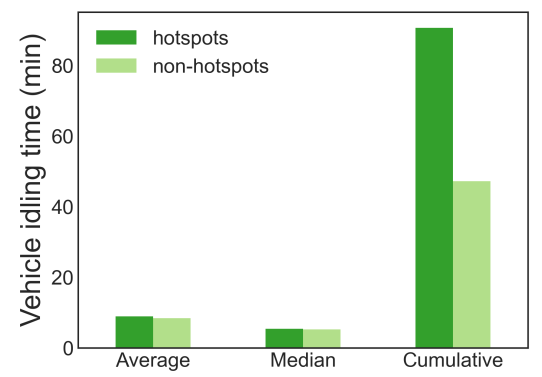

(d) Total vehicle idling time

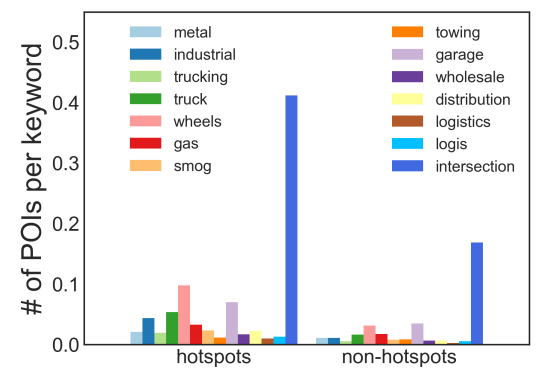

(b) POI name keywords

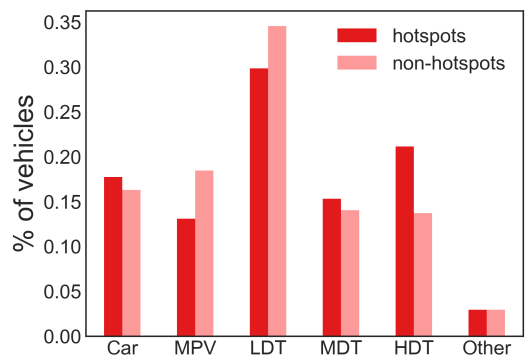

(e) Percent of vehicle types

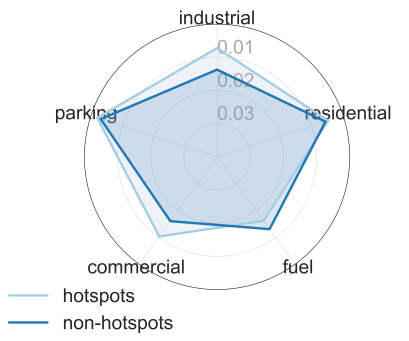

(c) Land use types

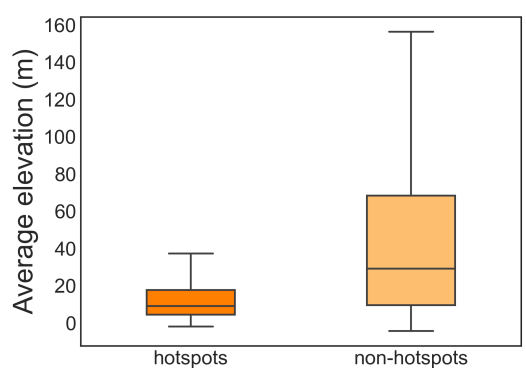

(f) Elevation

Figure 3: Comparison of hotspots vs. non-hotspots on various features including number of POIs per category, number of POIs per name keyword, distances to different land use types, vehicle idling time (MPV: multi-purpose vehicle, LDT, MDT, and HDT: light, medium, and high duty truck), and elevation.

\subsection{Hotspot Inference Results}

We use F1-score and AUC to evaluate the performance of hotspot inference. To deal with data imbalance issue (i.e., more non-hotspots than hotspots), we use resampling strategy which combines oversampling (i.e., SMOTE [4]) and undersampling approach to set an appropriate majority to minority class ratio. For hotspot inference, we train RandomForest classifier and apply 5-fold cross-validation. With all the extracted features, the model achieves an F1 score of 0.682 and AUC of 0.966 . Table 2 shows that using all the features outperforms every single feature set. Land use features perform the best, followed by vehicle idling, POI, and elevation features.

Table 2: Comparison of different feature sets.

\begin{tabular}{ccc}
\hline Feature sets (\# features) & F1-score & AUC \\
\hline All features (149) & 0.693 & 0.966 \\
Land use features (6) & 0.644 & 0.953 \\
Vehicle idling features (18) & 0.479 & 0.892 \\
POI features (122) & 0.466 & 0.855 \\
Elevation features (3) & 0.304 & 0.795 \\
\hline
\end{tabular}

\section{CONCLUSION}

We propose a two-phase framework to detect and infer air pollution hotspots. In the first phase, we design a two-step approach consists of local spike detection and sample-weighted clustering to detect hotspots using mobile sensing data. In the second phase, we leverage cross-domain urban data to extract features related to hotspots, and use them for hotspot inference. We evaluate this framework using real-world datasets. The evaluation results demonstrate the effectiveness of the proposed detection method and source features.

\section{ACKNOWLEDGMENTS}

We gratefully acknowledge Google for providing validated air quality datasets, and GeoTab for providing vehicle idling datasets. This work is supported by CyberSEES grant (NSF award \#1442971).

\section{REFERENCES}

[1] U.S. Environmental Protection Agency. [n.d.]. Research on near roadway and other near source air pollution. https://www.epa.gov/air-research/research-nearroadway-and-other-near-source-air-pollution.

[2] Joshua S. Apte, Kyle P. Messier, Shahzad Gani, Michael Brauer, Thomas W. Kirchstetter, Melissa M. Lunden, Julian D. Marshall, Christopher J. Portier, Roel C.H. Vermeulen, and Steven P. Hamburg. 2017. High-resolution air pollution mapping with Google Street View cars: Exploiting big data. Environmental Science \& Technology 51, 12 (2017), 6999-7008.

[3] Marie Chavent, Hervé Guegan, Vanessa Kuentz, Brigitte Patouille, and Jérôme Saracco. 2009. PCA-and PMF-based methodology for air pollution sources identification and apportionment. Environmetrics: The official journal of the International Environmetrics Society 20, 8 (2009), 928-942.

[4] Nitesh V Chawla, Kevin W Bowyer, Lawrence O Hall, and W Philip Kegelmeyer. 2002. SMOTE: synthetic minority over-sampling technique. Journal of artificial intelligence research 16 (2002), 321-357.

[5] Yizong Cheng. 1995. Mean shift, mode seeking, and clustering. IEEE transactions on pattern analysis and machine intelligence 17, 8 (1995), 790-799.

[6] Conor K Gately, Lucy R Hutyra, Scott Peterson, and Ian Sue Wing. 2017. Urban emissions hotspots: Quantifying vehicle congestion and air pollution using mobile phone GPS data. Environmental pollution 229 (2017), 496-504.

[7] Google. [n.d.]. Air Quality - Google Earth Outreach. https://www.google.com/ earth/outreach/special-projects/air-quality/.

[8] J Keith Ord and Arthur Getis. 1995. Local spatial autocorrelation statistics: distributional issues and an application. Geographical analysis 27, 4 (1995), 286-306.

[9] Chao Zhang, Keyang Zhang, Quan Yuan, Haoruo Peng, Yu Zheng, Tim Hanratty, Shaowen Wang, and Jiawei Han. 2017. Regions, periods, activities: Uncovering urban dynamics via cross-modal representation learning. In Proceedings of the 26th International Conference on World Wide Web. 361-370. 